\title{
An evaluation Paper An evaluation in vitro of the efficacy of nutlin-3 and topotecan in combination with ${ }^{177}$ Lu-DOTATATE for the treatment of neuroblastoma
}

\author{
Mathias Tesson ${ }^{1}$, Richa Vasan ${ }^{1}$, Andreas Hock², Colin Nixon ${ }^{2}$, Colin Rae ${ }^{1}$, Mark \\ Gaze $^{3}$ and Robert Mairs ${ }^{1}$ \\ ${ }^{1}$ Radiation Oncology, Institute of Cancer Sciences, Wolfson Wohl Translational Cancer Research Centre, University of Glasgow, \\ Bearsden, Glasgow, UK \\ ${ }^{2}$ Cancer Research UK Beatson Institute, Bearsden, Glasgow, UK \\ ${ }^{3}$ Department of Oncology, University College London Hospitals NHS Foundation Trust, London, UK \\ Correspondence to: Mathias Tesson, email: M.Tesson@beatson.gla.ac.uk \\ Keywords: DOTATATE; neuroblastoma; radiosensitisation; topotecan; nutlin-3
}

Received: August 09, $2017 \quad$ Accepted: May 28, $2018 \quad$ Published: June 26, 2018

Copyright: Tesson et al. This is an open-access article distributed under the terms of the Creative Commons Attribution License 3.0 (CC BY 3.0), which permits unrestricted use, distribution, and reproduction in any medium, provided the original author and source are credited.

\section{ABSTRACT}

Targeted radiotherapy of metastatic neuroblastoma using the somatostatin receptor (SSTR)-targeted octreotide analogue DOTATATE radiolabelled with lutetium-177 (177Lu-DOTATATE) is a promising strategy. This study evaluates whether its effectiveness may be enhanced by combination with radiosensitising drugs. The growth rate of multicellular tumour spheroids, derived from the neuroblastoma cell lines SK-N-BE(2C), CHLA-15 and CHLA-20, was evaluated following treatment with ${ }^{177}$ Lu-DOTATATE, nutlin-3 and topotecan alone or in combination. Immunoblotting, immunostaining and flow cytometric analyses were used to determine activation of p53 signalling and cell death. Exposure to ${ }^{177} \mathrm{Lu}$-DOTATATE resulted in a significant growth delay in CHLA-15 and CHLA-20 spheroids, but not in SK-N-BE(2c) spheroids. Nutlin-3 enhanced the spheroid growth delay induced by topotecan in CHLA-15 and CHLA-20 spheroids, but not in SK-N-BE(2c) spheroids. Importantly, the combination of nutlin-3 with topotecan enhanced the spheroid growth delay induced by $X$-irradiation or by exposure to ${ }^{177} \mathrm{Lu}$-DOTATATE. The efficacy of the combination treatments was p53-dependent. These results indicate that targeted radiotherapy of high risk neuroblastoma with ${ }^{177}$ Lu-DOTATATE may be improved by combination with the radiosensitising drugs nutlin-3 and topotecan.

\section{INTRODUCTION}

Neuroblastoma is a malignancy predominantly of infancy and young children. It originates most commonly in the adrenal glands [1] and affects about one hundred individuals per year in the UK (http://www. cancerresearchuk.org). About half of neuroblastomas are highly aggressive, as indicated by dissemination in children over one year of age or by adverse biological features such as amplification of the MYCN oncogene and by unresponsiveness to induction therapy or early relapse if a response is achieved. These high-risk neuroblastomas are responsible for $15 \%$ of paediatric cancer fatalities and new treatments are urgently needed [1]. Ninety percent of neuroblastoma tumours express the noradrenaline transporter (NAT). These can be treated with molecular radiotherapy using the iodine-131-radiolabelled noradrenaline analogue meta-iodobenzylguanidine ( $\left.{ }^{131} \mathrm{I}-\mathrm{mIBG}\right)$ [2]. Targeted radiotherapy of neuroblastoma using ${ }^{131} \mathrm{I}-\mathrm{mIBG}$ has produced encouraging longterm remission and palliation [3]. However, not all neuroblastoma tumours express NAT and resistance to ${ }^{131} \mathrm{I}$-mIBG therapy may occur [3]. This has encouraged the consideration of alternative radiopharmaceuticals for 
the targeted treatment of neuroblastoma. Somatostatin receptors (SSTRs) are overexpressed in human neuroblastomas [4-6] and the lutetium-177-radiolabelled somatostatin analogue DOTATATE $\left({ }^{177} \mathrm{Lu}-\mathrm{DOTATATE}\right)$ binds with high affinity to SSTRs, particularly subtype 2 (SSTR2). The efficacy and safety of ${ }^{177}$ Lu-DOTATATE therapy in neuroendocrine tumours have been reviewed elsewhere [7]. Its therapeutic application in neuroblastoma is appropriate particularly for patients whose tumours demonstrate good uptake of ${ }^{68} \mathrm{Ga}$-DOTATATE on PET/CT imaging, who do not have a positive ${ }^{123} \mathrm{I}-\mathrm{mIBG}$ diagnostic scan or whose tumours are unresponsive to, or relapse after, ${ }^{131} \mathrm{I}-\mathrm{mIBG}$ therapy.

The safe and successful treatment of children with neuroblastoma using ${ }^{177} \mathrm{Lu}$-DOTATATE was recently reported [8, 9]. However, it is expected that maximal therapeutic potency of targeted radiotherapy will be derived from its combination with radiosensitisers $[10,11]$. We previously showed that the topoisomerase I inhibitor topotecan enhanced ${ }^{131} \mathrm{I}-\mathrm{mIBG}$ efficacy in preclinical models [12]. Based on this study, the feasibility and tolerability of ${ }^{131} \mathrm{I}-\mathrm{mIBG}$ treatment in combination with topotecan were evaluated in children with metastatic neuroblastoma [13] and, subsequently, a prospective phase II study reported an overall response rate of $57 \%$ [14]. Finally, the multicentre randomised SIOPEN study EudraCT N $\mathrm{N}^{\circ}$ 2015-003130-27 will compare the effectiveness of ${ }^{131} \mathrm{I}-\mathrm{mIBG}$ in combination with topotecan to that of high dose thiotepa.

An alternative radiosensitising strategy is indicated by consideration of the role played by p53 in the response of neuroblastoma tumours to cytotoxic therapy. In neuroblastoma, the impairment of p53 signalling is seldom caused by inactivating mutations of the p53 gene $[15,16]$ but is more commonly due to upstream aberrations such as amplification of the p53 negative regulator MDM2 and inactivation of the MDM2 inhibitor p14(ARF) [17]. Genetic studies have indicated that the restoration of p53 activity has promising therapeutic potential $[18,19]$, notably through induction of apoptosis and senescence in tumour cells [20, 21]. For instance, the cis-imidazoline small molecule nutlin-3 binds to the p53-binding pocket of MDM2, stabilises p53 and reduces tumour cell viability [22]. It has also been shown to enhance the efficacy of targeted radiotherapy [23] and to sensitise tumour cells to radiation treatment via p53-mediated apoptosis [24] or senescence [25]. Interestingly, it has been reported that resistance of tumour cells to the cytotoxicity of p53 restoration could be overcome by radiation treatment [26]. Finally, the activation of the HIPK2 and p53 axis by nutlin-3 specifically in MYCN-amplified tumours sensitises tumours to apoptosis [27], thereby supporting that the strategy consisting of reactivating p53 in combination with radiotherapy is suitable for the treatment of patients with high risk neuroblastoma.
Topotecan has been shown to induce p53 expression $[28,29]$ and to synergise with MDM2 inhibitors in vitro and in vivo $[30,31]$. It has also been suggested that nutlin-3 may enhance the efficacy of chemotherapy $[32,33]$, notably through inhibition of multi-drug resistance protein 1 function [34]. Therefore, while others have demonstrated the benefit to be derived by the combination of nutlin-3 and topotecan, we hypothesised that this combination may sensitise neuroblastoma cells to ${ }^{177} \mathrm{Lu}$-DOTATATE targeted radiotherapy in a manner analogous to that of topotecan blended with ${ }^{131} \mathrm{I}$-mIBG treatment [12].

The aims of this study were first to characterise neuroblastoma cell lines with respect to their sensitivity to X-irradiation and ${ }^{177} \mathrm{Lu}$-DOTATATE as well as their ability to activate p53 signalling following treatment with nutlin-3, topotecan or X-irradiation; and second to assess whether the combination treatment consisting of topotecan and nutlin-3 sensitised neuroblastoma cells to X-irradiation and ${ }^{177} \mathrm{Lu}$-DOTATATE treatment and the relationship with activation of p53 signalling.

\section{RESULTS}

\section{Characterisation of the sensitivity of SK-N- BE(2c), CHLA-15 and CHLA-20 spheroids to ${ }^{177}$ Lu-DOTATATE treatment}

Uptake assays were performed in monolayers of various cell lines to determine their ability to concentrate ${ }^{177}$ Lu-DOTATATE intracellularly. The competitive inhibitor of binding to SSTR, octreotide, was used to determine whether binding of ${ }^{177} \mathrm{Lu}$-DOTATATE to SSTR was required for intracellular transport. There was significantly greater ${ }^{177} \mathrm{Lu}$-DOTATATE internalisation by SK-N-BE $(2 \mathrm{c})(P<0.001)$, CHLA-15 $(P<0.05)$ and CHLA-20 $(P<0.05)$ cells following exposure to ${ }^{177} \mathrm{Lu}-$ DOTATATE compared with exposure to ${ }^{177} \mathrm{Lu}$-DOTATATE in the presence of $1 \mu \mathrm{M}$ octreotide (Figure 1A). In contrast, UVW, PC12, SK-N-SH, SH-SY5Y and CHLA90 cells did not internalise ${ }^{177}$ Lu-DOTATATE (Figure 1A). Furthermore, there was a statistically significant, timedependent, accumulation of ${ }^{177}$ Lu-DOTATATE by SK-NBE(2c) $(P<0.001)$, CHLA-15 $(P<0.01)$ and CHLA-20 cells $(P<0.05)$, but not by UVW cells (Figure $1 \mathrm{~B})$. This observation is consistent with the expression of SSTR2 by SK-N-BE(2c), CHLA-15 and CHLA-20 cells, but not by UVW cells (Figure 1C). Immunoblotting analysis of SSTR2 revealed variation in the apparent molecular size of SSTR2, as indicated by a broad band (Figure 1C). This has been hypothesised previously to be due to posttranslational modifications [35, 36]. Finally, a growth delay mediated by $8 \mathrm{~h}$ exposure to ${ }^{177} \mathrm{Lu}$-DOTATATE was indicated by a 1.2-fold (not significant), 1.9-fold $(P<0.05)$ and 1.5 -fold $(P<0.01)$ decrease in AUC values in spheroids derived from SK-N-BE(2c), CHLA-15 and 
CHLA-20 cells, respectively (Figure 1D, Supplementary Figure 1A), suggesting that SK-N-BE(2c) spheroids were more resistant to ${ }^{177} \mathrm{Lu}$-DOTATATE than CHLA15 and CHLA-20 spheroids. This may be explained by their relative radiosensitivity. Indeed, SK-N-BE(2c) spheroids were significantly more resistant to 4 or 6 Gy X-irradiation than either CHLA-15 or CHLA-20 spheroids (Figure 1E, Supplementary Figure 1B). Moreover, the fold change in AUC in response to ${ }^{177} \mathrm{Lu}-$
DOTATATE treatment correlated with that obtained in response to X-irradiation $\left(r=0.347\right.$, adj $R^{2}=0.61$, $P=0.008$ ) (Figure 1F). Together, these results indicated that SK-N-BE(2c), CHLA-15 and CHLA-20 spheroids were suitable in vitro experimental models to assess SSTR2-targeted therapy. Furthermore, the sensitivity of spheroids to ${ }^{177} \mathrm{Lu}$-DOTATATE correlated with radiosensitivity, indicating that radiosensitisers may enhance the efficacy of ${ }^{177} \mathrm{Lu}$-DOTATATE treatment.
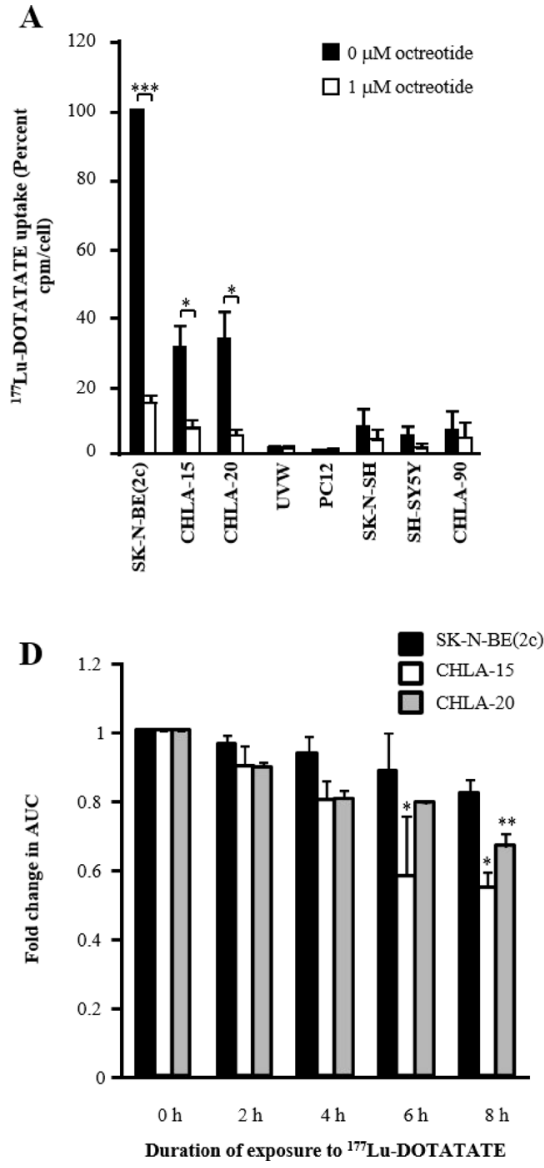

B

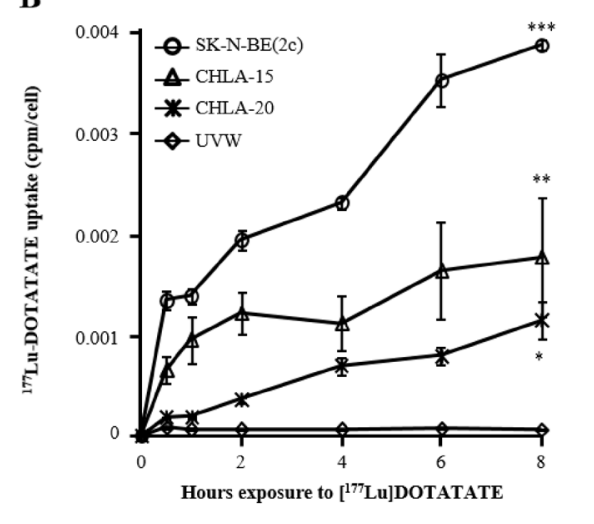

E

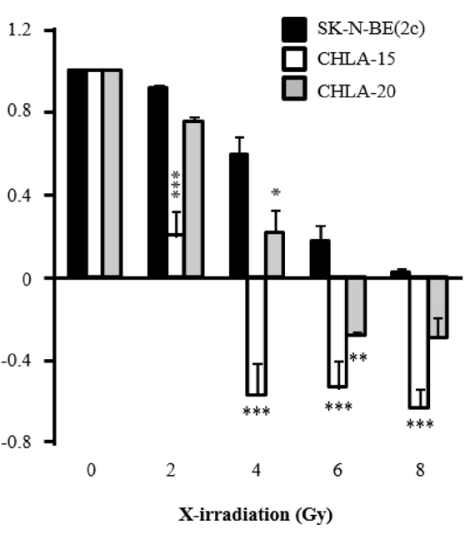

C

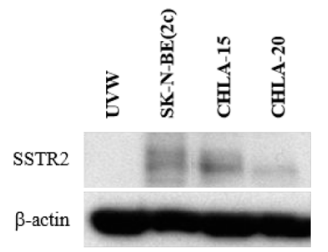

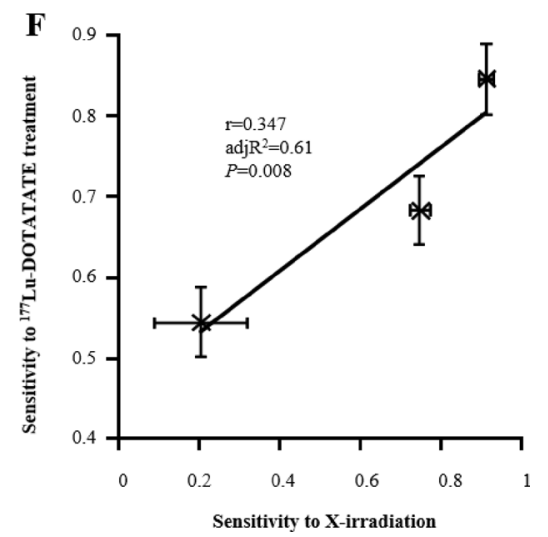

Figure 1: The effect of exposure of SSTR2-expressing cells to ${ }^{177} \mathrm{Lu}$-DOTATATE on its intracellular accumulation and spheroid growth delay. (A) SSTR-mediated uptake of ${ }^{177} \mathrm{Lu}$-DOTATATE by various cell lines was measured after $4 \mathrm{~h}$ incubation with $100 \mathrm{KBq} / \mathrm{ml}{ }^{177} \mathrm{Lu}$-DOTATATE in the presence or in the absence of $1 \mu \mathrm{M}$ octreotide. Data are means $\pm \mathrm{SEM}, n=3$. Paired-samples $t$-tests were performed to determine the significance of the reduction in uptake by octreotide. (B) The cytosolic accumulation of ${ }^{17}$ Lu-DOTATATE was measured over $8 \mathrm{~h}$ following treatment with $100 \mathrm{kBq} / \mathrm{ml}{ }^{177} \mathrm{Lu}$-DOTATATE. Data are means $\pm \mathrm{SEM}, n=3$. Bonferroni-corrected oneway ANOVA was performed. $P$-values indicate the significance of the difference between the mean uptake values at $8 \mathrm{~h}$ of SK-N-BE(2c), CHLA-15 and CHLA-20 cells in comparison with that of UVW cells. (C) The expression of SSTR2 was evaluated by immunoblotting in SK-N-BE(2c), CHLA-15 and CHLA-20 cells with UVW cells as negative control and $\beta$-actin as a loading control. (D) The spheroid growth delay induced by treatment of SK-N-BE(2c), CHLA-15 and CHLA-20 spheroids with $5 \mathrm{MBq} / \mathrm{ml}{ }^{177} \mathrm{Lu}$-DOTATATE for $8 \mathrm{~h}$ is reported as a decrease in AUC values. The radioactivity concentration $5 \mathrm{MBq} / \mathrm{ml}$ was chosen based on preliminary experiments. Data are mean $\pm \mathrm{SEM}, n=3$. One-way ANOVA with Bonferroni correction was performed. $P$-values indicate the significance of the difference in AUC values in comparison with that of untreated spheroids for each cell line. (E) The radiosensitivity of SK-N-BE(2c), CHLA-15 and CHLA-20 spheroids. Data are mean \pm SEM, $n=3$. One-way ANOVA with Bonferroni correction was performed. At each radiation dose, $P$-values indicate the significance of the difference in AUC values in comparison with that of SK-N-BE(2c) spheroids. (F) The AUC values of SK-N-BE(2c), CHLA-15 and CHLA-20 spheroids following treatment with $5 \mathrm{MBq} / \mathrm{ml}{ }^{177} \mathrm{Lu}$-DOTATATE for $8 \mathrm{~h}$ correlated with AUC values obtained following treatment with 2 Gy X-irradiation. Data are mean $\pm \mathrm{SEM}, n=3$. In all panels, one symbol indicates $P<0.05$, two symbols indicate $P<0.01$ and three symbols indicate $P<0.001$. 


\section{Characterisation of the sensitivity of SK-N-BE(2c), CHLA-15 and CHLA-20 cells to treatment with nutlin-3 and topotecan alone or in combination}

In SK-N-BE(2c) cells, p53 expression was not increased in response to $\mathrm{X}$-irradiation despite phosphorylation at serine 15 (Figure 2), a typical marker for p53 activation. The absence of p21 $1^{\text {Cip//Wafl }}$ (p21) expression indicated that the transcriptional activity of $\mathrm{p} 53$ was impaired at the $\mathrm{p} 21$ gene promoter in SK-N$\mathrm{BE}(2 \mathrm{c})$ cells in response to $\mathrm{X}$-irradiation (Figure 2). This conclusion is supported by the previously reported detection, in SK-N-BE(2c) cells, of mutations in the DNAbinding domain of p53 [37]. In contrast, in CHLA-15 and CHLA-20 cells, the increase in p53 expression in response to $\mathrm{X}$-irradiation was associated with its activation by phosphorylation at serine 15 and expression of $\mathrm{p} 21$ (Figure 2). This observation indicated that, in response to X-irradiation, p53 is an active transcription factor at the p21 gene promoter in CHLA-15 and CHLA-20 cells. Endogenous levels of p53 were higher in SK-N-BE(2c) cells than in CHLA-15 and CHLA-20 cells (Figure 2), perhaps reflecting a compensatory mechanism resulting from the impairment of p53 transcriptional activity.

Next, we determined the effect of exposure of SKN-BE(2c), CHLA-15 and CHLA-20 cells to nutlin-3 and topotecan on p53 expression and PARP cleavage, a marker of caspase activity which is a hallmark of apoptotic cell death. This analysis was carried out in cellular monolayers (Figure 3A) as well as in spheroids (Figure 3B). In SK-N-BE(2c) cells, combination treatment consisting of nutlin-3 with topotecan resulted in apoptotic cell death. This was probably due to topotecan alone since treatment with nutlin-3 as a single agent did not cause apoptosis
(Figure 3A and 3B). In contrast, treatment of CHLA-15 and CHLA-20 cells with nutlin-3 and topotecan alone or in combination resulted in increased p53 expression and PARP cleavage (Figure 3A and 3B). Furthermore, an increase in BAX expression was observed following treatment of CHLA-20 cells with nutlin-3 and topotecan alone or in combination (data not shown). Together, these results indicated that treatment with nutlin-3 induced p53 expression and apoptotic cell death in CHLA-15 and CHLA-20 cells which harbour transcriptionally active p53, whereas topotecan induced apoptotic cell death irrespective of the p53 status of SK-N-BE(2c), CHLA-15 and CHLA-20 cells.

The effect of nutlin-3 and topotecan on cell cycle distribution was evaluated in SK-N-BE(2c), CHLA-15 and CHLA-20 cells grown as cellular monolayers (Figure 3C). The statistical analysis of the changes in the proportion of cells in Sub-G1, a measure of apoptosis induction, G0-G1, $\mathrm{S}$ and G2-M are summarised in Table 1. In SK-N-BE(2c) cells, nutlin-3 and topotecan alone or in combination did not cause a significant increase in sub-G1 population. This observation was contrasted by the significant increase in the sub-G1 population following nutlin-3 and topotecan alone or in combination in CHLA-15 and CHLA-20 cells (Figure 3C, Table 1). Topotecan caused a significant decrease in the proportion of cells in S and $\mathrm{G} 2-\mathrm{M}$ in CHLA-15 and CHLA-20 but not in SK-N-BE(2c) cells (Figure 3C, Table 1). The combination of nutlin-3 and topotecan induced, in CHLA-15 and CHLA-20 but not in SK-N-BE(2c) cells, a significant decrease in the G2-M population in comparison with the effect of nutlin-3 or topotecan as single agents (Figure 3C, Table 1). Together, these results indicated that, in the $\mathrm{p} 53$ functional cell lines CHLA-15 and CHLA-20, the depletion of cells in S and

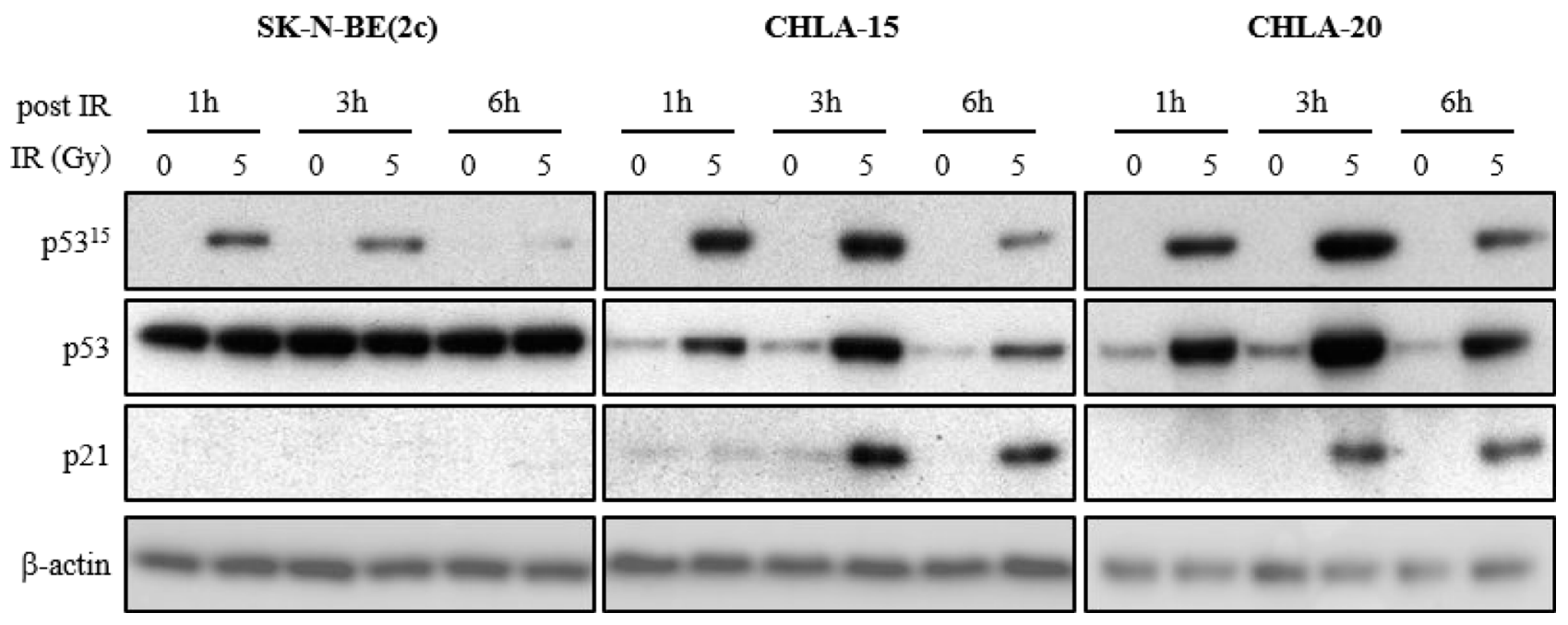

Figure 2: The effect of X-irradiation on p53 signalling in SK-N-BE(2c), CHLA-15 and CHLA-20 cells. The expression of $\mathrm{p} 53$ phosphorylated at serine $15\left(\mathrm{p} 53^{15}\right)$, total p53 and p21 in SK-N-BE(2c), CHLA-15 and CHLA-20 cells was evaluated by immunoblotting 1, 3 and $6 \mathrm{~h}$ following X-irradiation with $5 \mathrm{~Gy} \cdot \mathrm{p} 53^{15}$ is a marker of p53 activation by upstream kinases, p21 is a marker of 53 transcriptional activity and $\beta$-actin is used as a loading control. 
G2-M phases of the cell cycle by treatment with nutlin-3 and topotecan was probably due to apoptotic cell death in these particular phases of the cell cycle.

Finally, as spheroids grow, their heterogeneous internal morphology is characterised by a hypoxic, nondividing and dying core encircled by a proliferating outer layer [23]. The growth delay experiments were designed to treat the spheroids when they were $100 \mu \mathrm{m}$ in diameter and to monitor them until they grew to a diameter of $1 \mu \mathrm{m}$. The exposure of $100 \mu \mathrm{m}$ diameter spheroids to the combination treatment consisting of nutlin-3 with topotecan resulted in a significantly increased growth delay in CHLA15 and CHLA-20 spheroids in comparison with single agent treatments (Figure 3D, Supplementary Figure 2). In contrast, there was no significant enhancement of topotecan-induced growth delay in SK-N-BE(2c) spheroids by combination with nutlin-3 (Figure 3D).

Together, these results suggested that the combination treatment consisting of nutlin-3 and topotecan may be more potent in tumours capable of activating p53 signalling. To evaluate this conjecture, we performed p53 gene expression knockdown in SH-SY5Y

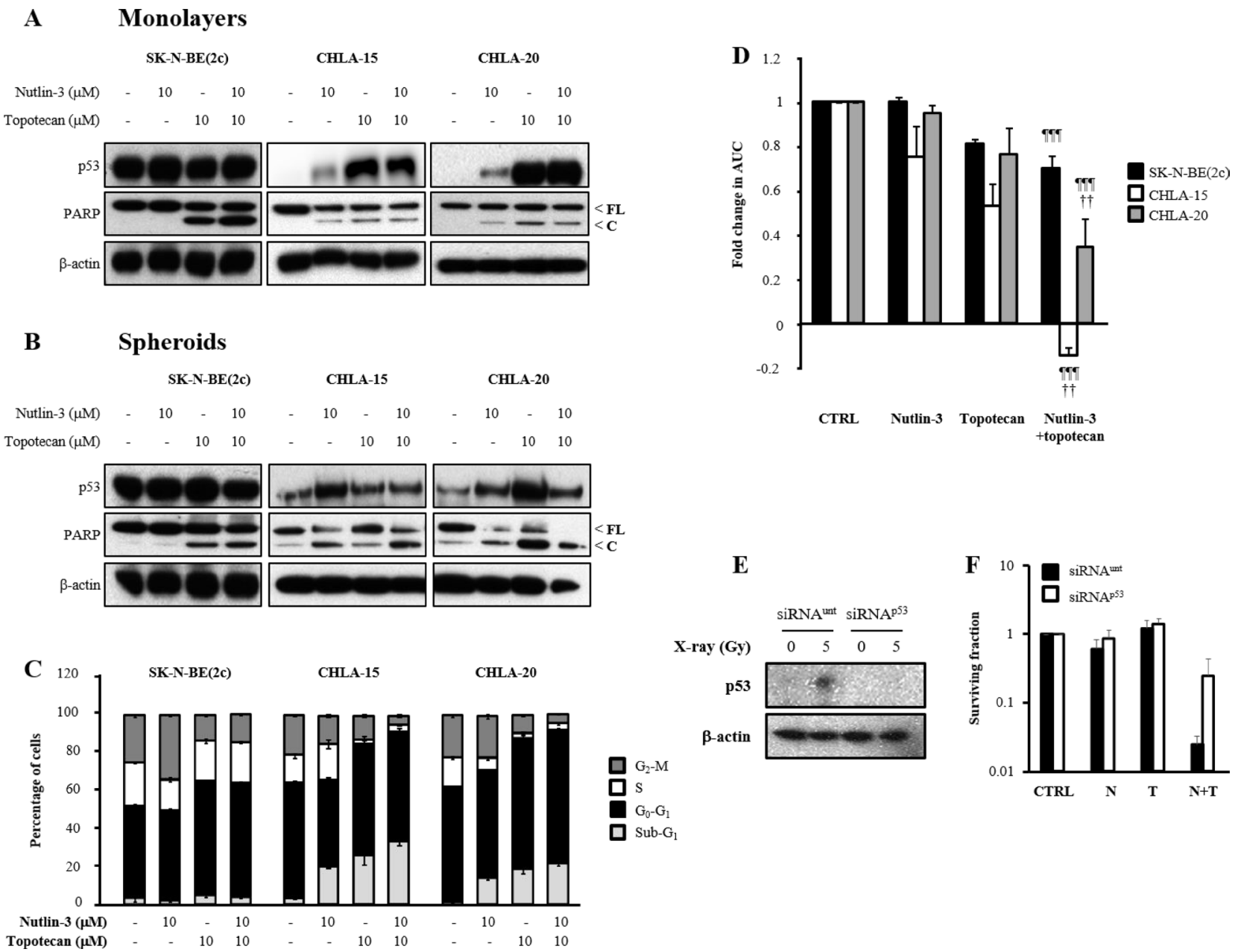

Figure 3: The effect of nutlin-3 and topotecan alone or in combination on the induction of p53 signalling, cell cycle arrest and spheroid growth delay. The expression of p53, full-length (FL) and cleaved (C) PARP was evaluated by immunoblotting $6 \mathrm{~h}$ in monolayers (A) or $24 \mathrm{~h}$ in spheroids (B) following treatment of SK-N-BE(2c), CHLA-15 and CHLA-20 cells with nutlin-3 and topotecan alone or in combination. (C) The distribution of SK-N-BE(2c), CHLA-15 and CHLA-20 cells throughout the phases of the cell cycle was evaluated in monolayers by flow cytometry analysis of propidium iodide-stained cells following treatment for $12 \mathrm{~h}$ with nutlin-3 and topotecan alone or in combination. (D) SK-N-BE(2c), CHLA-15 and CHLA-20 spheroids were treated with $10 \mu \mathrm{M}$ nutlin-3 and $10 \mu \mathrm{M}$ topotecan alone or in combination for $24 \mathrm{~h}$. The median AUC values resulting from each treatment were statistically compared with each other using Mann-Whitney pairwise comparisons. The symbol *indicates a comparison with untreated control, the symbol "indicates a comparison with nutlin-3 alone and the symbol indicates a comparison with topotecan alone. One symbol indicates $P<0.05$, two symbols indicate $P<0.01$ and three symbols indicate $P<0.001$. (E) Immunoblotting was used to determine the ability of p53-targeted siRNA $\left(\right.$ siRNA $\left.^{\mathrm{p} 53}\right)$ or untargeted siRNA (siRNA ${ }^{\text {unt }}$ ) to abrogate the increase in p53 expression level following X-irradiation. SH-SY5Y cells were

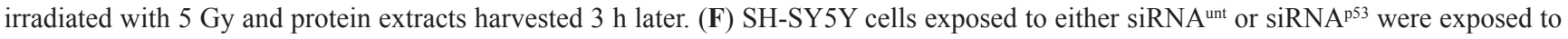
$200 \mathrm{nM}$ nutlin-3 and $5 \mathrm{nM}$ topotecan alone or in combination and the clonogenic cell kill was determined. 
Table 1: Cell cycle analysis after treatment of cells with nutlin-3 and topotecan alone or in combination

\begin{tabular}{|c|c|c|c|c|}
\hline \multicolumn{5}{|c|}{ SK-N-BE(2c) } \\
\hline & Sub-G1 & G0-G1 & $\mathbf{S}$ & G2-M \\
\hline Control & $3.68 \pm 1.64$ & $46.6 \pm 0.93$ & $22.1 \pm 0.25$ & $23.58 \pm 0.74$ \\
\hline $10 \mu \mathrm{M}$ nutlin-3 & $2.44 \pm 0.75$ & $44.8 \pm 1.10$ & $15.6 \pm 1.24^{*}$ & $32.2 \pm 0.56^{* * *}$ \\
\hline $10 \mu \mathrm{M}$ topotecan & $5.31 \pm 0.98$ & $58.6 \pm 0.52^{*}$ & $20.6 \pm 1.19$ & $13.1 \pm 0.50^{* * *}$ \\
\hline Nutlin-3 + toptecan & $4.02 \pm 0.17$ & $58.5 \pm 0.85^{\text {ศ19 }}$ & $21.0 \pm 0.53^{\pi / \pi}$ & $14.2 \pm 0.29$ ตศศ \\
\hline \multicolumn{5}{|c|}{ CHLA-15 } \\
\hline & Sub-G1 & G0-G1 & $\mathbf{S}$ & G2-M \\
\hline Control & $3.16 \pm 0.60$ & $58.8 \pm 3.67$ & $14.3 \pm 2.81$ & $20.6 \pm 1.13$ \\
\hline $10 \mu \mathrm{M}$ nutlin-3 & $19.8 \pm 1.70^{* *}$ & $44.7 \pm 3.65^{*}$ & $18.8 \pm 5.34$ & $14.6 \pm 1.60^{* * *}$ \\
\hline $10 \mu \mathrm{M}$ topotecan & $25.8 \pm 12.2^{* * *}$ & $58.2 \pm 10.6$ & $1.73 \pm 0.33^{* * *}$ & $12.8 \pm 1.90^{* * *}$ \\
\hline Nutlin-3 + toptecan & $33.1 \pm 5.36$ & $56.9 \pm 5.04^{\pi}$ & $3.35 \pm 0.59^{999}$ & $4.57 \pm 0.74^{949+\dagger \dagger}$ \\
\hline \multicolumn{5}{|c|}{ CHLA-20 } \\
\hline & Sub-G1 & G0-G1 & $\mathbf{S}$ & G2-M \\
\hline Control & $1.73 \pm 0.26$ & $59.7 \pm 0.76$ & $15.6 \pm 0.67$ & $21.6 \pm 0.84$ \\
\hline $10 \mu \mathrm{M}$ nutlin-3 & $14.4 \pm 1.23^{* * *}$ & $56.5 \pm 0.81$ & $6.54 \pm 0.53^{* * *}$ & $21.9 \pm 1.15$ \\
\hline $10 \mu \mathrm{M}$ topotecan & $18.8 \pm 1.99^{* * *}$ & $68.7 \pm 1.78^{* * *}$ & $2.78 \pm 0.63^{* * *}$ & $9.41 \pm 0.51^{* * *}$ \\
\hline Nutlin-3 + toptecan & $22.2 \pm 1.40^{\Uparrow \pi}$ & $69.3 \pm 1.05^{\text {ตศศ }}$ & $3.49 \pm 0.65^{\natural}$ & $4.79 \pm 0.20^{9199 \dagger \dagger}$ \\
\hline
\end{tabular}

Propidium iodide-stained cells were analyzed using flow cytometry to determine cell cycle distribution $24 \mathrm{~h}$ following treatment of SK-N-BE(2c), CHLA-15 and CHLA-20 cells with $10 \mu \mathrm{M}$ nutlin-3 and topotecan alone or in combination. One-way ANOVA with Bonferroni correction was performed. ", indicates a comparison with untreated control, "indicates a comparison with nutlin-3 alone, "indicates a comparison with topotecan alone. One symbol indicates $P<0.05$, two symbols indicate $P<0.01$ and three symbols indicate $P<0.001$.

cells followed by evaluation of the clonogenic cell kill induced by the combination of nutlin-3 and topotecan. We hypothesised that transfection of SK-N-BE(2c) cells with a p53-expressing vector would not result in a functional p53 axis due to the endogenous expression of mutant $\mathrm{p} 53$. In response to $\mathrm{X}$-irradiation, there was an increase in p53 expression in SH-SY5Y cells exposed to untargeted siRNA but not in SH-SY5Y cells treated with p53-targeted siRNA (Figure 3E). In response to combination treatment consisting of nutlin-3 with topotecan, there was a 10-fold increase in clonogenic cell growth in p53-silenced SHSY5Y cells in comparison with SH-SY5Y cells exposed to untargeted siRNA (Figure 3F). This observation indicated that cytotoxicity resulting from the combination of nutlin-3 with topotecan depends on a functional p53 signalling. In agreement, it has previously been reported that nutlin-3 and topotecan interact in a synergistic manner in cell lines with wild-type p53 [30, 31].

The sensitivity of tumours to cytotoxic treatment is known to depend on their size which influences drug penetration and resistance to treatment due to heterogeneous areas of quiescence and/or hypoxia [38]. Accordingly, we evaluated the effect of spheroid size on the cytotoxicity of nutlin-3 and topotecan. SK-N-BE(2c) and CHLA-20 spheroids were allowed to grow for 3 weeks before exposure to nutlin-3 and topotecan as single agents. CHLA-15 spheroids were not recovered because of extensive disaggregation caused by the treatments. Similarly, combination treatments of SK-N-BE(2c) and CHLA-20 spheroids resulted in disaggregation and loss of spheroid morphology which precluded harvesting for immunohistochemistry. In CHLA-20, but not in SK$\mathrm{N}-\mathrm{BE}(2 \mathrm{c})$ spheroids, nutlin-3 and topotecan treatments increased expression of p53 and cleaved caspase 3, a marker of apoptotic cell death (Figure 4). In SK-N-BE(2c) spheroids, only topotecan treatment increased expression of cleaved caspase 3 (Figure 4). Importantly, caspase 3 cleavage was observed throughout the spheroid, indicating that the phenomenon of drug resistance due to poor tissue penetration is unlikely in micrometastases. Furthermore, the induction, by nutlin-3 and topotecan, of apoptotic cell death throughout spheroids, suggested that, as well as targeted radiopharmaceuticals, nutlin-3 and topotecan are suitable treatment modalities for malignant lesions in the class size of micrometastases.

\section{Enhancement of ${ }^{177}$ Lu-DOTATATE-induced spheroid growth delay by nutlin-3 and topotecan}

Both topotecan and nutlin-3 have been shown to enhance targeted radiotherapy and to be radiosensitisers [12, $23,24]$. Therefore we evaluated the effect of nutlin-3 and 
topotecan alone or in combination on the spheroid growth delay induced by ${ }^{177} \mathrm{Lu}$-DOTATATE. The combination of 10 $\mu \mathrm{M}$ of nutlin-3 and topotecan sterilised CHLA-15 spheroids (Figure 3D, Supplementary Figure 2). Consequently, in order to observe modulation of the spheroid growth delay by combination with ${ }^{177}$ Lu-DOTATATE, CHLA15 spheroids were also treated with an alternative, lower dosage of nutlin-3 and topotecan. Combined treatment with $5 \mu \mathrm{M}$ nutlin-3 and $5 \mu \mathrm{M}$ topotecan resulted in a significantly increased growth delay $(P<0.01)$ in CHLA15 spheroids in comparison with single agent treatments, without sterilisation (Supplementary Figure 3).

Firstly, we determined the spheroid growth delay resulting from treatment with nutlin-3 and topotecan alone
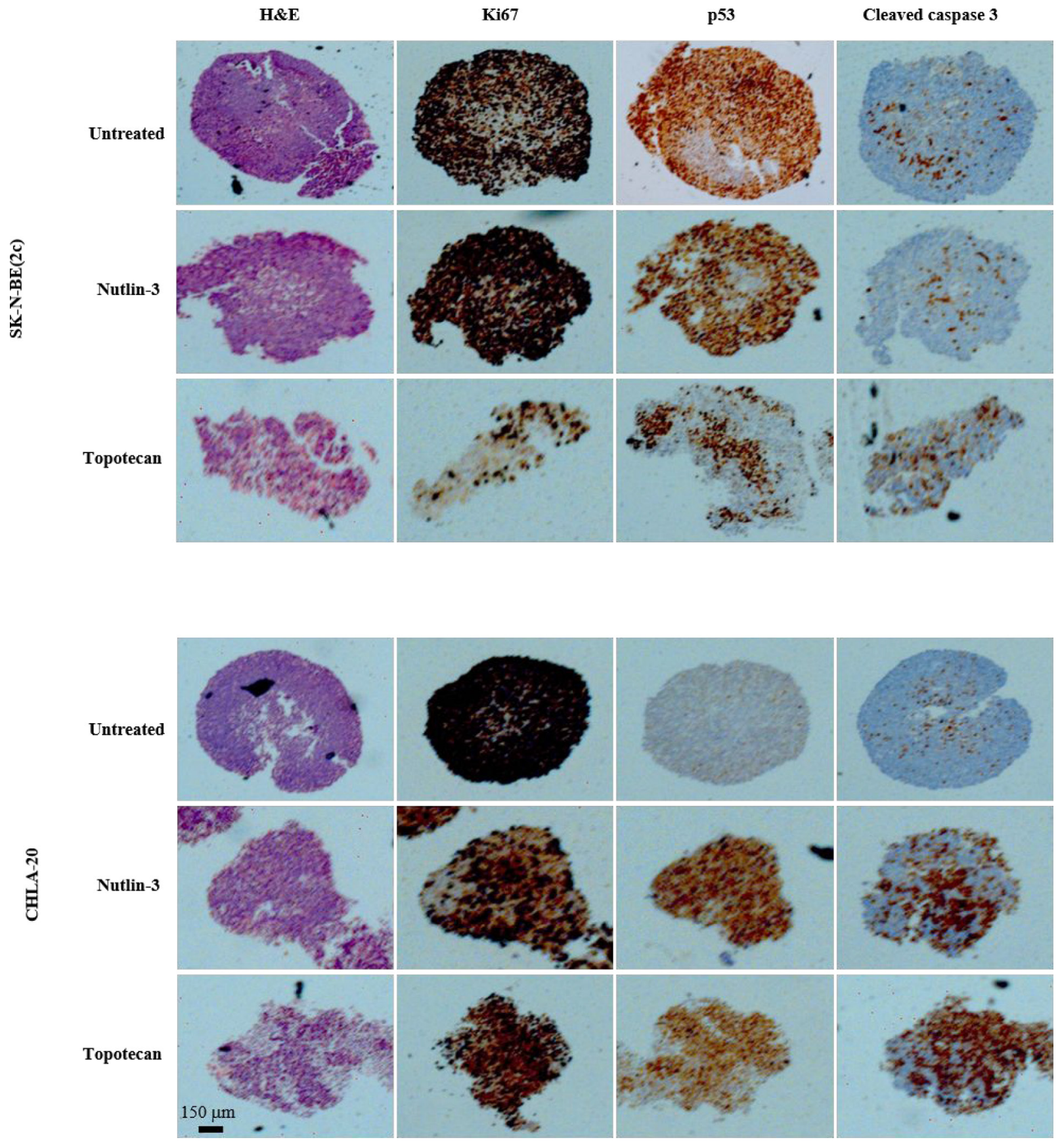

Figure 4: The effect of nutlin-3 and topotecan on cellular proliferation, p53 expression and apoptotic cell death in SK-N-BE(2c) and CHLA-20 spheroids. The markers of histological organisation (haematoxylin and eosin, H\&E), proliferation (Ki67), p53 expression and apoptotic cell death (cleaved caspase 3) were detected by immunohistochemistry in SK-N-BE(2c) and CHLA-20 spheroids. The spheroids were allowed to grow for 3 weeks to reach $1 \mathrm{~mm}$ in diameter prior exposure to $10 \mu \mathrm{M}$ nutlin-3 or $10 \mu \mathrm{M}$ topotecan for $24 \mathrm{~h}$. 
or in combination with ${ }^{177}$ Lu-DOTATATE. Although not statistically significant, topotecan and the combination treatment consisting of nutlin-3 with topotecan enhanced the spheroid growth delay induced by ${ }^{177} \mathrm{Lu}$-DOTATATE (Figure 5A, Supplementary Figure 4A). Moreover, inhibition of proliferation was more prominent in CHLA15 and CHLA-20 spheroids, which harbour a functional p53 signalling pathway, than in SK-N-BE(2c) spheroids which do not (Figure 5A, Supplementary Figure 4A).

Secondly, the effect of nutlin-3 and topotecan alone or in combination on X-irradiation-induced spheroid growth delay was evaluated to determine whether radiosensitisation could explain the enhancement of ${ }^{177} \mathrm{Lu}$-DOTATATE efficacy. Similarly to the combination treatments with ${ }^{177}$ Lu-DOTATATE, topotecan and the combination treatment consisting of nutlin-3 with topotecan enhanced the spheroid growth delay induced by X-irradiation although statistical significance was not reached (Figure 5B, Supplementary Figure 4B). Furthermore, growth inhibition was more prominent in CHLA-15 and CHLA20 than in SK-N-BE(2c) spheroids. For instance, nutlin-3 enhanced X-irradiation-induced spheroid growth delay to a greater extent in CHLA-20 spheroids than in SK-N-BE(2c) spheroids (Figure 5B). This observation was consistent with the enhanced increase in p53 and cleaved PARP expression following combination treatment consisting of nutlin-3 and $\mathrm{X}$-irradiation in comparison with nutlin-3 alone or $\mathrm{X}$-irradiation alone in CHLA-20 cells but not in SK-N$\mathrm{BE}(2 \mathrm{c})$ cells (Figure 5C). Similarly, the triple combination consisting of nutlin-3, topotecan and X-irradiation increased growth delay in CHLA-15 spheroids to a greater extent than in SK-N-BE(2c) spheroids (Figure 5B). PARP cleavage and p53 expression may already be maximal after treatment with topotecan and no further increase was possible following triple combination treatment consisting of nutlin-3, topotecan and X-irradiation (Figure 5C).

\section{DISCUSSION}

Multicellular tumour spheroids are well-established models of prevascular micrometastases that provide a means of studying the intratumoural distribution of therapeutic agents and of determining the effect of cytotoxic agents. They have previously been used extensively in targeted therapy research to investigate diffusion gradients of targeting agents, to assess efficacies of alternative modalities, to evaluate microdosimetry and to provide experimental model systems for testing hypotheses [11]. In this study, using multicellular tumour spheroids, we show that the radiosensitising combination consisting of nutlin-3 and topotecan enhanced the spheroid growth delay induced by ${ }^{177} \mathrm{Lu}$-DOTATATE. This effect was more prominent in spheroids composed of cells which harbour functional p53 signalling and constitutes an appropriate therapeutic option to target malignant lesions the size of micrometastases.
Due to the cytotoxic and cytostatic effects of somatostatin, the expression of SSTRs has been found to inversely correlate with $\mathrm{MYCN}$ amplification and to positively correlate with survival of patients with differentiated neuroblastomas [39-41]. However, these studies also found a subset of neuroblastoma tumours with MYCN amplification or classified as stage III-IV that were characterised by levels of SSTRs expression equivalent to tumours with a better prognosis [39-41]. Therefore, ${ }^{177} \mathrm{Lu}$-DOTATATE therapy is suitable for high risk neuroblastoma in a subset of patients with a positive ${ }^{68} \mathrm{Ga}$-DOTATATE diagnostic scan.

We found that SSTR2 expression levels were greater in SK-N-BE(2c) than in CHLA-15 and CHLA20 cells (Figure $1 C$ ). This result was corroborated by the significant increased uptake of ${ }^{177}$ Lu-DOTATATE by SKN-BE(2c) cells than by CHLA-15 and CHLA-20 cells (Figure 1B). Although, it has recently been reported that SK-N-BE(2c) cells express lower levels of SSTR2 than CHLA-15 and CHLA-20 cells, this study did not report the outcome of a functional assay [42]. Furthermore, in our study, we observed that the spheroid growth delay induced by ${ }^{177} \mathrm{Lu}$-DOTATATE increased with increasing duration of exposure. This observation might be explained by the intracellular trafficking of SSTRs following agonist-induced internalisation [43]. For instance, it has been shown that SSTR2 is transported intracellularly and recycled back to the membrane within $1 \mathrm{~h}$ following octreotide stimulation [44]. The recycling of SSTR2 back to the membrane within $1 \mathrm{~h}$ could allow the binding and internalisation of a second ${ }^{177}$ Lu-DOTATATE molecule. Thus, the longer the exposure to ${ }^{177} \mathrm{Lu}$-DOTATATE, the greater the internalised amount of ${ }^{177} \mathrm{Lu}$-DOTATATE. In conjunction with the prolonged retention of ${ }^{177} \mathrm{Lu}-$ DOTATATE within tumours [45], the time-dependent cytotoxicity of ${ }^{177} \mathrm{Lu}$-DOTATATE can be seen as a positive aspect of this therapy.

Recently, microtubule polymerisation has been suggested to be a target of topotecan, thereby challenging the opinion that topotecan's cytotoxicity is due solely to topoisomerase I inhibition [46]. In our study, we found that $10 \mu \mathrm{M}$ topotecan as a single agent specifically killed cells in $\mathrm{S}$ and G2-M phases of the cell cycle (Table 1). However, it has been reported that this concentration may be too high to inhibit microtubule polymerisation [47]. Therefore, it is expected that the cellular depletion in the G2-M phase of the cell cycle is due to $\mathrm{S}$ phase-specific cell death rather than to interference with microtubule polymerisation during mitosis. Since S phase cells are radioresistant, radiosensitisation by topotecan may be explained by $\mathrm{S}$ phase-specific cell death. Furthermore, the combination of nutlin-3 with topotecan caused a significant decrease in the proportion of cells in G2-M (Table 1). The depletion of cells in $\mathrm{S}$ phase by topotecan alone and the depletion of cells in G2-M by the combination of nutlin-3 with topotecan were only significant in CHLA-15 and CHLA- 
20 cells - which are capable of activating p53 signalling - but not in SK-N-BE(2c) cells. This observation can be explained by the known roles of p53 in cell death and the activation of cell cycle checkpoints in S and G2 [47].

It has been shown that the p53 status of a tumour cell is a determinant of sensitivity to topotecan although this is subject to debate. For instance, it has been reported that p53 triggers the proteasomal degradation of topoisomerase I leading to resistance to topotecan [48] and that topotecan treatment is more potent in p53-deficient cells because of downregulation of XIAP and survivin [49]. In contrast, it has been suggested that the p53 status of glioma cell lines does not predict sensitivity to topotecan [50]. Importantly, in SK-N-BE(2c) spheroids, unable to activate p53 signalling, treatment with nutlin-3 in combination with topotecan did not reverse the enhancement of spheroid growth delay induced by topotecan. This observation suggests that although the combination of MDM2 inhibitors and topotecan is likely to provide more benefit for patients whose tumours are p53 functional, this combination treatment may not result in a lower efficacy in patients with p53 non-functional tumours. Therefore, if MDM2 inhibitors are found to be clinically safe, these molecules may be given in addition to topotecan regardless to the p53 status of the patient's tumour, thereby eliminating the need for the development of biomarkers of p53 signalling.
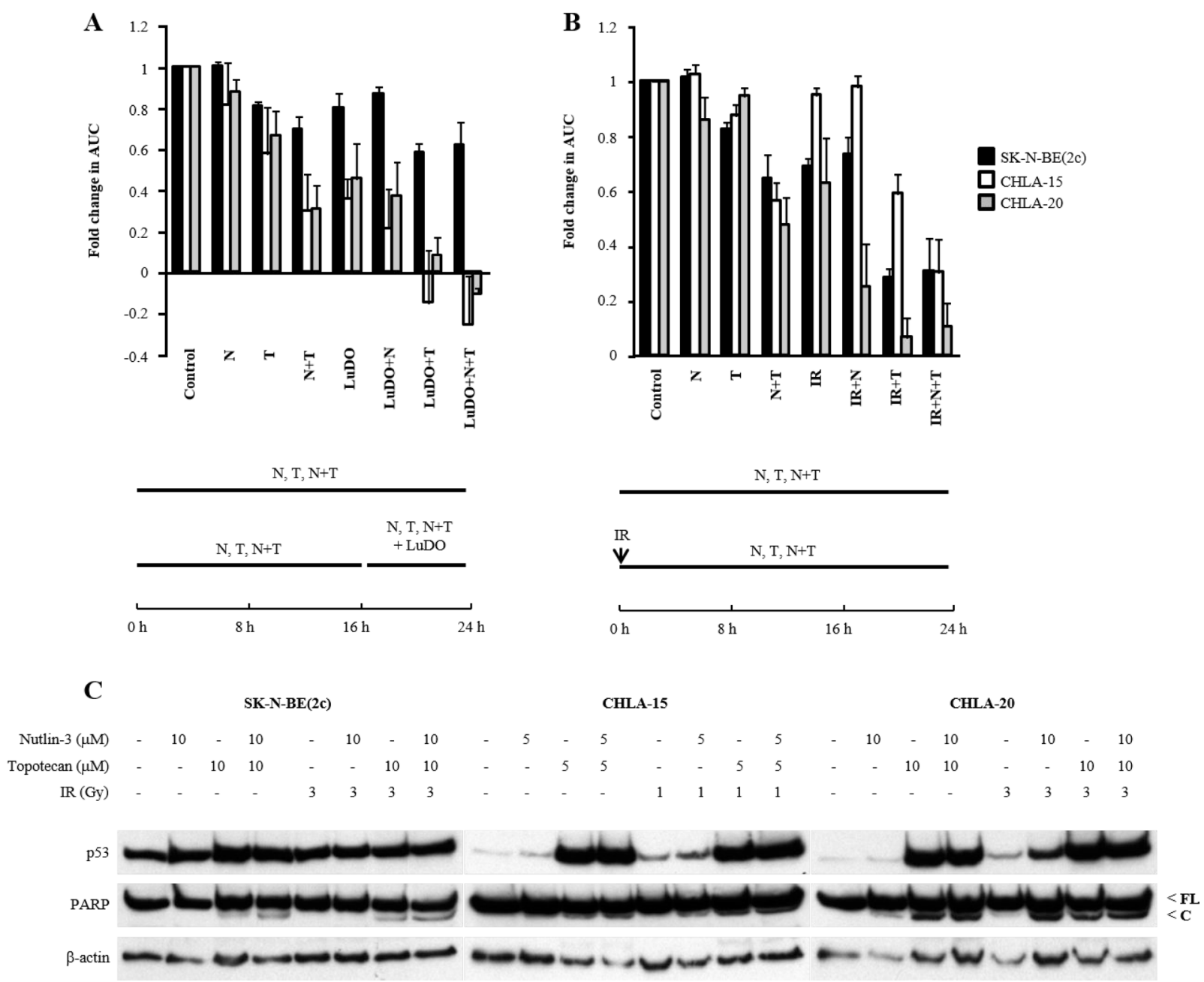

Figure 5: The effect of nutlin-3 and topotecan alone or in combination on the spheroid growth delay induced by X-irradiation or ${ }^{177}$ Lu-DOTATATE. (A) SK-N-BE(2c) and CHLA-20 spheroids were treated with $10 \mu \mathrm{M}$ nutlin-3 (N) and $10 \mu \mathrm{M}$ topotecan (T) for $24 \mathrm{~h}$. CHLA-15 spheroids were treated with $5 \mu \mathrm{M}$ nutlin-3 and $5 \mu \mathrm{M}$ topotecan. ${ }^{177}$ Lu-DOTATATE (LuDO) treatment consisted of exposure for $8 \mathrm{~h}$ to $5 \mathrm{MBq} / \mathrm{ml}$. The schedule of administrations of nutlin-3, topotecan and ${ }^{177} \mathrm{Lu}$-DOTATATE is shown below the histogram. (B) SK-N-BE(2c), CHLA-15 and CHLA-20 spheroids were pre-treated with nutlin-3 and topotecan alone or in combination for $24 \mathrm{~h}$. The spheroids were irradiated (IR) with $3 \mathrm{~Gy}, 1 \mathrm{~Gy}$ and $3 \mathrm{~Gy}$, respectively, at the start of exposure to nutlin-3 and topotecan. The schedule of administrations of nutlin-3, topotecan and IR is shown below the histogram. (C) The expression of p53, full-length (FL) and cleaved (C) PARP was evaluated by immunoblotting $6 \mathrm{~h}$ following treatment of SK-N-BE(2c), CHLA-15 and CHLA-20 monolayers with nutlin-3, topotecan and X-irradiation alone or in combination. (A, B) The median AUC values resulting from each treatment were statistically compared with each other using Mann-Whitney pairwise comparisons. However, statistical significance was not reached. 
The selection of patients who will most likely benefit from therapy is a major area of medical research. The levels of MDM2 expression have been reported to be prognostic and predictive biomarkers $[51,52]$. Specifically, MDM2 expression or the presence of the MDM2 SNP309 polymorphism, which causes increased expression of the MDM2 gene, correlated negatively with outcome in patients with mesothelioma [53, 54]. Furthermore, MDM2 expression or the presence of the MDM2 SNP309 genetic variant correlated positively with sensitivity to chemotherapeutic agents including topoisomerase I inhibitors [53]. These studies suggest that MDM2 amplification and the MDM2 polymorphism SNP309 have prognostic value and may serve as predictive biomarkers of chemotherapy efficacy.

Recently, p53-independent MDM2 functions have been described with respect to possible adverse sideeffects in patients [54]. Specifically, MDM2 binds to the retinoblastoma tumour suppressor $(\mathrm{Rb})$ and promotes its degradation [54]. Besides its tumour suppressor activity, $\mathrm{Rb}$ also regulates muscle development and myoblast proliferation and differentiation [54]. It has been shown that nutlin-3 treatment, which results in increased MDM2 levels due to $\mathrm{p} 53$ activation, leads to $\mathrm{Rb}$ degradation and decreased levels of myoblast proliferation and differentiation markers, suggesting potential clinical complications [54]. However, the strategy consisting of reactivating p53 is currently being tested in various phase I clinical trials of cancer in order to assess safety and tolerability [55]. For instance, the inhibitor of the p53-MDM2 interaction, MK-8242, was investigated in 47 patients with p53-wild-type solid tumours [56]. Its safety and tolerability at dosage producing clinical responses provided a rationale for further testing in a phase II clinical trial [56]. In another study, responses have been observed following administrations of MDM2 inhibitors with acceptable toxicity profiles [57]. Our present study, and others [30-33], suggest that the optimal use of nutlin-3 may be in combination with alternative therapies. Since ${ }^{177} \mathrm{Lu}$-DOTATATE concentrates in malignant lesions, the enhancement of chemotherapy efficacy by the combination with nutlin-3 only occurs in targeted cells, thus sparing normal tissues and reducing the gravity of adverse effects.

The goal of combination therapy is the potentiation of tumour-specific cytotoxicity at lower dosages of therapeutic agents while avoiding an increase in the risk of adverse effects in healthy tissues. The blending of drugs with non-overlapping mechanisms of action that function in concert to reactivate p53 may be a strategy to achieve this goal. Firstly, MDMX is structurally related to MDM2 and reduces p53 activity [18, 19]. The strategy consisting of dual inhibition of the p53-MDM2 and p53MDMX interactions has shown promise in pre-clinical testing [58]. Secondly, the p53 gene product Wip1/ PPM1D is a phosphatase which dephosphorylates and deactivates $\mathrm{p} 53$ [59]. The combination of nutlin-3 with Wip1 inhibition has been shown to be synergistic and to potently reactivate p53 signalling [60]. Interestingly, it has been shown that the novel Wip1 inhibitor GSK2830371 reduced the growth of neuroblastoma xenografts in mice and enhanced the cytotoxicity caused by topoisomerase II inhibitors [61]. In the light of these observations, the radiosensitising properties of the nutlin-3 and topotecan combination may be safely enhanced further by dual inhibition of p53-MDM2 and p53-MDMX interactions or by inhibition of Wip1.

In summary, this study indicates that the efficacy of ${ }^{177} \mathrm{Lu}$-DOTATATE treatment of children with high risk neuroblastoma may be enhanced by the combination with the radiosensitising drugs nutlin-3 and topotecan. The precise dose-scheduling which will be optimal remains to be determined. Interestingly, it was recently reported that a single injection of ${ }^{177} \mathrm{Lu}$-DOTATATE in mice bearing CHLA-15 xenografts did not induce a significant longlasting growth delay [42], indicating that combination of ${ }^{177} \mathrm{Lu}$-DOTATATE with radiosensitisers may be beneficial. Further experiments in animal models of neuroblastoma are therefore warranted to facilitate the clinical development of the combination of nutlin-3 and topotecan with ${ }^{177} \mathrm{Lu}$-DOTATATE. This strategy holds promise as another component in the multimodality arsenal for the treatment of children with high risk neuroblastoma.

\section{MATERIALS AND METHODS}

\section{Cell culture}

The neuroblastoma cell lines CHLA-90, CHLA15 and CHLA-20 were obtained from the Children's Oncology Group and maintained in Iscove's Modified Dulbecco's medium supplemented with $2 \mathrm{mM}$ L-glutamine, 1\% (v/v) insulin, 1\% (v/v) transferrin, $1 \%(\mathrm{v} / \mathrm{v})$ selenium and $20 \%(\mathrm{v} / \mathrm{v})$ foetal bovine serum (FBS, Autogen Bioclear, UK) at $37^{\circ} \mathrm{C}$ in a $5 \% \mathrm{CO}_{2}$ atmosphere. The neuroblastoma cell lines SK-N-BE(2c), SK-N-SH and SH-SY-5Y and the phaeochromocytoma cell line PC12 were obtained from the American Tissue Culture Collection. SK-N-BE(2c) cells were maintained in Dulbecco's Modified Eagle medium (DMEM) supplemented with $15 \%$ (v/v) FBS, 2 mM L-glutamine and $1 \%(\mathrm{v} / \mathrm{v})$ non-essential amino acids at $37^{\circ} \mathrm{C}$ in a $5 \%$ $\mathrm{CO}_{2}$ atmosphere. PC12, SH-SY5Y and SK-N-SH cells were maintained in DMEM medium supplemented with $10 \%(\mathrm{v} / \mathrm{v}) \mathrm{FBS}$ and $2 \mathrm{mM} \mathrm{L}$-glutamine at $37^{\circ} \mathrm{C}$ in a $5 \%$ $\mathrm{CO}_{2}$ atmosphere. The glioma cell line UVW was described previously [62]. UVW cells were maintained in Minimum Essential Medium supplemented with 10\% (v/v) FBS and $2 \mathrm{mM}$ L-glutamine at $37^{\circ} \mathrm{C}$ in a $5 \% \mathrm{CO}_{2}$ atmosphere. Unless otherwise stated, all reagents used for cell culture were purchased from ThermoFisher Scientific (UK). 


\section{Silencing of p53 expression}

The transfection of siRNAs was performed with Lullaby ${ }^{\circledR}$ siRNA transfection reagent according to the manufacturer's protocol (OZ Biosciences, Marseille, France). The sense sequence of the p53-targeted siRNA was GAC UCC AGU GGU AAU CUA CUU Dharmacon (Lafayette, Colorado). The siGENOME non-targeting siRNA pool \#2 from Dharmacon (Lafayette, Colorado) was used as a negative control. Following exposure to $124 \mathrm{nM}$ siRNAs for $72 \mathrm{~h}$, the cells were suspended by treatment with trypsin and seeded in triplicates at 2,000 cells per well in 6-well plates. Once attached, the cells were exposed to $200 \mathrm{nM}$ nutlin-3 and $5 \mathrm{nM}$ topotecan alone and in combination and incubated at $37^{\circ} \mathrm{C}$ in $5 \% \mathrm{CO}_{2}$ to allow for colony formation. Once the colonies comprised more than 50 cells, the medium was removed and the colonies were fixed in $50 \%$ methanol $(\mathrm{v} / \mathrm{v})$ in PBS and stained with $1 \%(\mathrm{v} / \mathrm{v})$ crystal violet in PBS before counting.

\section{${ }^{177}$ Lu-DOTATATE uptake assay}

${ }^{177}$ Lu-DOTATATE was obtained from Advanced Accelerator Applications, France. The specific activity was $37 \mathrm{GBq} / \mathrm{mg}$. Cellular monolayers were incubated for 2, 4, 6 and $8 \mathrm{~h}$ at $37^{\circ} \mathrm{C}$ in a $5 \% \mathrm{CO}_{2}$ atmosphere in culture medium containing $100 \mathrm{kBq} / \mathrm{ml}{ }^{177} \mathrm{Lu}$-DOTATATE in the presence or in the absence of $1 \mu \mathrm{M}$ octreotide, a competitive inhibitor of binding to SSTR. The concentration of octreotide and the radioactivity concentration of ${ }^{177}$ Lu-DOTATATE were selected based on our preliminary investigation (data not shown). They were then washed three times in PBS. The radioactivity retained in the cells was extracted with $10 \%$ $(w / v)$ trichloroacetic acid and measured using a $\gamma$-counter (Canberra Packard, UK). The number of cells was counted using a haemocytometer in a separate identical culture and ${ }^{177} \mathrm{Lu}$-DOTATATE uptake was expressed as counts per minute $(\mathrm{cpm})$ per cell.

\section{Spheroid initiation}

Spheroids were obtained using the liquid overlay technique [63]. Cellular monolayers were trypsinised and reseeded at a cellular density of 160,000 cells.cm ${ }^{-2}$ in ultralow attachment flasks (Corning, Netherlands). Spheroids formed within 2 days.

\section{Treatment of spheroids and growth curve analysis}

In order to assess the efficacy of radiopharmaceuticals combined with radiosensitisers, we selected the spheroid growth delay. A three-dimensional culture system is required to adequately represent therapeutic effects upon micrometastases (a major aim of targeted radionuclides) and because radionuclides are toxic to tumours not only because their decay particles inflict damage in targeted cells but also because cross-fire irradiation to neighbouring, untargeted cells contributes significantly to efficacy. Finally, spheroid growth was monitored for 21 days - a period long enough to observe at least a 100 -fold increase in volume. Twenty-one days is similar to the time period required for the formation of colonies in clonogenic assay. Single agent treatments consisted of exposure of the spheroids to ${ }^{177} \mathrm{Lu}$-DOTATATE for $8 \mathrm{~h}$ or to nutlin-3 and topotecan for $24 \mathrm{~h}$. Combination treatments consisted of exposure of the spheroids to nutlin-3 and/ or topotecan for $16 \mathrm{~h}$ followed by exposure to nutlin-3 and/or topotecan in combination with ${ }^{177} \mathrm{Lu}$-DOTATATE for $8 \mathrm{~h}$. At the end of treatments, the spheroids were washed three times in PBS. Then, those of approximately $100 \mu \mathrm{m}$ in diameter were manually selected and individually transferred into ultra-low attachment plates for monitoring (Corning, Netherlands). For every spheroid, two orthogonal diameters, $\mathrm{d}_{\max }$ and $\mathrm{d}_{\min }$, were measured twice per week using the image analysis software ImageJ (National Institute of Health, Bethesda, MD, USA) and the volume, $\mathrm{V}$, was calculated using: $\mathrm{V}=\pi \times \mathrm{d}_{\max }$ $\times \mathrm{d}_{\min }{ }^{2} / 6,000,000$ [64]. The spheroid volume, at various time points after the initiation of treatment, was computed as the quotient of volume at time $t$ divided by volume at time zero $\left(\mathrm{V} / \mathrm{V}_{0}\right)$. In order to evaluate the effect of treatment over the course of an experiment, the area under the $\log \mathrm{V} / \mathrm{V}_{0}$ versus time curve (AUC) was calculated for individual spheroids using trapezoidal approximation. Spheroid growth delay caused by a treatment was indicated by a reduction of the AUC value in comparison with that of the untreated spheroids.

\section{X-ray irradiation}

Monolayers or spheroids were irradiated using an RS225 irradiator (Xstrahl, UK) at room temperature with $195 \mathrm{kV} / 10 \mathrm{mAX}$-rays producing a dose rate of $1.64 \mathrm{~Gy} / \mathrm{min}$ at a distance of $30 \mathrm{~cm}$.

\section{Statistical analysis of spheroid growth delay}

The distribution of AUC values was not normal, as indicated by the Shapiro-Wilk test. Therefore, nonparametric Kruskal-Wallis testing was used to determine whether experimental data indicated a significant level of difference between the medians of the groups. If the $P$-value corresponding to the Kruskal-Wallis test was less than 0.05, the Mann-Whitney test was used for pairwise comparisons. The comparison of single agent treatment with combination treatment was characterised by the following features. Firstly, in order to demonstrate enhancement of ${ }^{177} \mathrm{Lu}$-DOTATATE-induced spheroid growth delay, the observed effect in response to combination treatment of a radiosensitiser with ${ }^{177} \mathrm{Lu}$ DOTATATE had to be greater than that induced by ${ }^{177} \mathrm{Lu}-$ DOTATATE or the radiosensitiser alone. Secondly, an absence of enhancement of ${ }^{177} \mathrm{Lu}$-DOTATATE-induced 
growth delay could be due to insufficient radiosensitiser dosage. Therefore, the evaluation of the modification of the effect of ${ }^{177} \mathrm{Lu}$-DOTATATE involved a family of four pairwise comparisons: radiosensitiser versus untreated control, ${ }^{177}$ Lu-DOTATATE versus untreated control, radiosensitiser $+{ }^{177} \mathrm{Lu}$-DOTATATE versus radiosensitiser and radiosensitiser $+{ }^{177} \mathrm{Lu}$-DOTATATE versus ${ }^{177} \mathrm{Lu}-$ DOTATATE. To compensate for multiple pairwise comparisons, Bonferroni correction was applied. In order to retain the criterion $P<0.05$, the level of significance of each pairwise comparison was set to 0.0125 .

\section{Immunoblotting}

Antibodies against SSTR2 and $\beta$-actin were obtained from Abcam, UK. The antibody against p53 was obtained from Santa-Cruz, US. Antibodies against p53 phosphorylated at serine 15 and PARP were obtained from New England Biolabs, UK. The antibody against p21 was obtained from BD Pharmingen, UK. Whole cellular protein extracts were resolved in reducing and denaturing conditions by sodium dodecyl sulphate polyacrylamide gel electrophoresis. Proteins were transferred on to polyvinylidene fluoride (PVDF) Immobilon-P membranes (Millipore, UK). Membranes were blocked with 7.5\% $(\mathrm{w} / \mathrm{v})$ milk for $2 \mathrm{~h}$ prior to incubation with the primary antibodies overnight at $4^{\circ} \mathrm{C}$. Membranes were then washed and incubated at room temperature for $1 \mathrm{~h}$ with horseradish peroxidase-conjugated secondary antimouse (Cell Signalling, UK) or anti-rabbit antibody (Cell Signalling, UK) to enable chemiluminescent detection using ECL (ThermoFisher Scientific, UK).

\section{Cell cycle analysis}

SK-N-BE(2c), CHLA-15 and CHLA-20 cellular monolayers were exposed for $12 \mathrm{~h}$ to $10 \mu \mathrm{M}$ nutlin-3 and $10 \mu \mathrm{M}$ topotecan alone or in combination. After $12 \mathrm{~h}$, the cells were harvested by trypsinisation and fixed in $70 \%(\mathrm{v} / \mathrm{v})$ ethanol at $-20^{\circ} \mathrm{C}$. The cells were stained with $10 \mu \mathrm{g} / \mathrm{ml}$ propidium iodide and $200 \mu \mathrm{g} / \mathrm{ml}$ RNAse A for at least 10 min prior to analysis using a FACSCalibur flow cytometry system (BD Biosciences, UK). Flow cytometric data were quantified using FlowJo 7.6.5 software.

\section{Immunocytochemistry}

After treatment, the spheroids were fixed in $10 \%$ neutral formalin (Cellpath, UK) for $24 \mathrm{~h}$ at $4^{\circ} \mathrm{C}$. The spheroids were agitated and gently mixed before being carefully placed at the centre of biopsy paper (Leica, UK). The biopsy paper was then folded over twice and placed into the tissue processing cassette for processing through 70, 90, 95\% (v/v) alcohol, 100\% (v/v) ethanol, xylene and molten wax. The solid wax that may have formed was removed by placing the unfolded biopsy paper containing the spheroids onto a hot plate. Using heated forceps, the spheroids were then transferred from the biopsy paper into a tissue embedding mold containing molten wax. A tissue cassette was finally placed onto the embedding mold and left to harden. Once hardened, the paraffin wax block was removed and $4 \mu \mathrm{m}$ sections were obtained using a Finesse microtome (ThermoFischer, UK). Spheroid sections were de-waxed in xylene and re-hydrated by successive immersions in graded alcohol and tap water. Endogenous peroxidase activity was quenched using peroxidase-blocking solution (Dako, UK). Heat-induced antigen retrieval was performed in a $10 \mathrm{mM}$ sodium citrate, $0.05 \%$ (v/v) Tween 20, pH6 buffer at $98^{\circ} \mathrm{C}$ for 25 minutes using a pre-treatment module (Dako, UK). The sections were then washed using Tris-buffered Tween before being exposed to antiKi67 (ThermoFisher Scientific, UK), anti-p53 (Leika, UK) or anti-cleaved caspase 3 (Cell Signalling, UK) antibodies. Species appropriate secondary antibodies (Dako EnVision, UK) were applied for $30 \mathrm{~min}$ followed by 3,3'-diaminobenzidine tetrahydrochloride for $10 \mathrm{~min}$. The reaction was terminated with deionized water for $1 \mathrm{~min}$. The sections were then counterstained with Gills haematoxylin and the nuclei blued using Scott's tap water. The sections were then dehydrated through graded alcohol, cleared in xylene and mounted with a glass coverslip using DPX mountant for microscopy.

\section{Author contributions}

MT: study design, data acquisition, data analysis and interpretation, statistical analysis and drafting of the manuscript. RV: data acquisition. $\mathrm{AH}$ : data acquisition and drafting of the manuscript. $\mathrm{CN}$ : data acquisition. CR: data interpretation and drafting of the manuscript. $\mathrm{MG}$ : data interpretation and drafting of the manuscript. RM: data interpretation and drafting of the manuscript.

\section{ACKNOWLEDGMENTS}

The authors are grateful to Dr Matt Aldridge for supplying ${ }^{177}$ Lu-DOTATATE.

\section{CONFLICTS OF INTEREST}

The authors report no financial disclosure, funding or conflicts of interest.

\section{FUNDING}

This work was supported by the joint Action Medical Research and Neuroblastoma UK grant GN2196. MNG is supported by the National Institute for Health Research University College London Hospitals Biomedical Research Centre. 


\section{REFERENCES}

1. Cole KA, Maris JM. New strategies in refractory and recurrent neuroblastoma: translational opportunities to impact patient outcome. Clin Cancer Res. 2012; 18:2423-28.

2. Wilson JS, Gains JE, Moroz V, Wheatley K, Gaze MN. A systematic review of 131I-meta iodobenzylguanidine molecular radiotherapy for neuroblastoma. Eur J Cancer. 2014; 50:801-15.

3. Matthay KK, Yanik G, Messina J, Quach A, Huberty J, Cheng SC, Veatch J, Goldsby R, Brophy P, Kersun LS, Hawkins RA, Maris JM. Phase II study on the effect of disease sites, age, and prior therapy on response to iodine-131metaiodobenzylguanidine therapy in refractory neuroblastoma. J Clin Oncol. 2007; 25:1054-60.

4. Georgantzi K, Tsolakis AV, Stridsberg M, Jakobson A, Christofferson R, Janson ET. Differentiated expression of somatostatin receptor subtypes in experimental models and clinical neuroblastoma. Pediatr Blood Cancer. 2011; 56:584-9.

5. Maggi M, Baldi E, Finetti G, Franceschelli F, Brocchi A, Lanzillotti R, Serio M, Camboni MG, Thiele CJ. Identification, characterization, and biological activity of somatostatin receptors in human neuroblastoma cell lines. Cancer Res. 1994; 54:124-33.

6. O'Dorisio MS, Chen F, O'Dorisio TM, Wray D, Qualman SJ. Characterization of somatostatin receptors on human neuroblastoma tumors. Cell Growth Differ. 1994; 5:1-8.

7. Brabander T, Teunissen JJ, Van Eijck CH, Franssen GJ, Feelders RA, de Herder WW, Kwekkeboom DJ. Peptide receptor radionuclide therapy of neuroendocrine tumours. Best Pract Res Clin Endocrinol Metab. 2016; 30:103-14.

8. Gains JE, Bomanji JB, Fersht NL, Sullivan T, D'Souza D, Sullivan KP, Aldridge M, Waddington W, Gaze MN. 177Lu-DOTATATE molecular radiotherapy for childhood neuroblastoma. J Nucl Med. 2011; 52:1041-7.

9. Kong G, Hofman MS, Murray WK, Wilson S, Wood P, Downie P, Super L, Hogg A, Eu P, Hicks RJ. Initial Experience With Gallium-68 DOTA-Octreotate PET/CT and Peptide Receptor Radionuclide Therapy for Pediatric Patients With Refractory Metastatic Neuroblastoma. J Pediatr Hematol Oncol. 2016; 38:87-96.

10. Gaze MN, Gains JE, Walker C, Bomanji JB. Optimization of molecular radiotherapy with [131I]-meta Iodobenzylguanidine for high-risk neuroblastoma. Q J Nucl Med Mol Imaging. 2013; 57:66-78.

11. Mairs RJ, Boyd M. Preclinical assessment of strategies for enhancement of metaiodobenzylguanidine therapy of neuroendocrine tumors. Semin Nucl Med. 2011; 41:334- 44.

12. McCluskey AG, Boyd M, Ross SC, Cosimo E, Clark AM, Angerson WJ, Gaze MN, Mairs RJ. [I-131]metaldobenzylguanidine and topotecan combination treatment of tumors expressing the noradrenaline transporter. Clin Cancer Res. 2005; 11:7929-37.
13. Gaze MN, Chang YC, Flux GD, Mairs RJ, Saran FH, Meller ST. Feasibility of dosimetry-based high-dose 131I-metaiodobenzylguanidine with topotecan as a radiosensitiser in children with metastatic neuroblastoma. Cancer Biother Radiopharm. 2005; 20:195-9.

14. Kraal KC, Tytgat GA, van Eck-Smit BL, Kam B, Caron HN, van Noesel M. Upfront treatment of high-risk neuroblastoma with a combination of $131 \mathrm{I}-\mathrm{mIBG}$ and topotecan. Pediatr Blood Cancer. 2015; 62:1886-91.

15. Hosoi G, Hara J, Okamura T, Osugi Y, Ishihara S, Fukuzawa M, Okada A, Okada S, Tawa A. Low frequency of the p53 gene mutations in neuroblastoma. Cancer. 1994; 73:3087-93.

16. Imamura J, Bartram CR, Berthold F, Harms D, Nakamura H, Koeffler HP. Mutation of the p53 gene in neuroblastoma and its relationship with N-myc amplification. Cancer Res. 1993; 53:4053-8.

17. Carr-Wilkinson J, O'Toole K, Wood KM, Challen CC, Baker AG, Board JR, Evans L, Cole M, Cheung NK, Boos J, Kölher G, Leuschner I, Pearson AD, et al. High Frequency of p53/MDM2/p14ARF Pathway Abnormalities in Relapsed Neuroblastoma. Clin Cancer Res. 2010; 16:1108-18.

18. Ribeiro CJA, Rodrigues CMP, Moreira R, Santos MMM. Chemical variations on the p53 reactivation theme. Pharmaceuticals (Basel). 2016; 9:E25.

19. Selivanova G. Wild type $\mathrm{p} 53$ reactivation: from lab bench to clinic. FEBS Lett. 2014; 588:2628-38.

20. Ventura A, Kirsch DG, McLaughlin ME, Tuveson DA, Grimm J, Lintault L, Newman J, Reczek EE, Weissleder R, Jacks T. Restoration of p53 function leads to tumour regression in vivo. Nature. 2007; 445:661-5.

21. Xue W, Zender L, Miething C, Dickins RA, Hernando E, Krizhanovsky V, Cordon-Cardo C, Lowe SW. Senescence and tumour clearance is triggered by p53 restoration in murine liver carcinomas. Nature. 2007; 445:656-60.

22. Vassilev LT, Vu BT, Graves B, Carvajal D, Podlaski F, Filipovic Z, Kong N, Kammlott U, Lukacs C, Klein C, Fotouhi $\mathrm{N}$, Liu EA. In vivo activation of the p53 pathway by smallmolecule antagonists of MDM2. Science. 2004; 303:844-8.

23. Tesson M, Rae C, Nixon C, Babich JW, Mairs RJ. Preliminary evaluation of prostate-targeted radiotherapy using (131) I-MIP-1095 in combination with radiosensitising chemotherapeutic drugs. J Pharm Pharmacol. 2016; 68:912-21.

24. Cao C, Shinohara ET, Subhawong TK, Geng L, Kim KW, Albert JM, Hallahan DE, Lu B. Radiosensitization of lung cancer by nutlin, an inhibitor of murine double minute 2 . Mol Cancer Ther. 2006; 5:411-7.

25. Lehmann BD, McCubrey JA, Jefferson HS, Paine MS, Chappell WH, Terrian DM. A dominant role for p53dependent cellular senescence in radiosensitization of human prostate cancer cells. Cell Cycle. 2007; 6:595-605.

26. Martins CP, Brown-Swigart L, Evan GI. Modeling the therapeutic efficacy of $\mathrm{p} 53$ restoration in tumors. Cell. 2006; 127:1323-34. 
27. Petroni M, Veschi V, Prodosmo A, Rinaldo C, Massimi I, Carbonari M, Dominici C, McDowell HP, Rinaldi C, Screpanti I, Frati L, Bartolazzi A, Gulino A, et al. MYCN sensitizes human neuroblastoma to apoptosis by HIPK2 activation through a DNA damage response. Mol Cancer Res. 2011; 9:67-77.

28. Daoud SS, Munson PJ, Reinhold W, Young L, Prabhu VV, Yu Q, LaRose J, Kohn KW, Weinstein JN, Pommier Y. Impact of p53 knockout and topotecan treatment on gene expression profiles in human colon carcinoma cells: a pharmacogenomic study. Cancer Res. 2003; 63:2782-93.

29. Li DD, Sun T, Wu XQ, Chen SP, Deng R, Jiang S, Feng GK, Pan JX, Zhang XS, Zeng YX, Zhu XF. The inhibition of autophagy sensitises colon cancer cells with wild-type p53 but not mutant p53 to topotecan treatment. PLoS One. 2012; 7:e45058.

30. Chen L, Rousseau RF, Middleton SA, Nichols GL, Newell DR, Lunec J, Tweddle DA. Pre-clinical evaluation of the MDM2-p53 antagonist RG7388 alone and in combination with chemotherapy in neuroblastoma. Oncotarget. 2015; 6:10207-21. https://doi.org/10.18632/oncotarget.3504.

31. De Lange J, Ly LV, Lodder K, Verlaan-de Vries M, Teunisse AF, Jager MJ, Jochemsen AG. Synergistic growth inhibition based on small-molecule p53 activation as treatment for intraocular melanoma. Oncogene. 2012; 31:1105-16.

32. Deben C, Wouters A, Op de Beeck K, van Den Bossche J, Jacobs J, Zwaenepoel K, Peeters M, Van Meerbeeck J, Lardon F, Rolfo C, Deschoolmeester V, Pauwels P. The MDM2-inhibitor Nutlin-3 synergizes with cisplatin to induce p53 dependent tumor cell apoptosis in non-small cell lung cancer. Oncotarget. 2015; 6:22666-79. https://doi. org/10.18632/oncotarget.4433.

33. Voon YL, Ahmad M, Wong PF, Husaini R, Ng WT, Leong CO, Lane DP, Khoo AS. Nutlin-3 sensitizes nasopharyngeal carcinoma cells to cisplatin-induced cytotoxicity. Oncol Rep. 2015; 34:1692-1700.

34. Chen L, Zhao Y, Halliday GC, Berry P, Rousseau RF, Middleton SA, Nichols GL, Del Bello F, Piergentili A, Newell DR, Lunec J, Tweddle DA. Structurally diverse MDM2-p53 antagonists act as modulators of MDR-1 function in neuroblastoma. Br J Cancer. 2014; 111:716-25.

35. Pfeiffer M, Koch T, Schroder H, Klutzny M, Kirscht S, Kreienkamp HJ, Höllt V, Schulz S. Homo- and heterodimerization of somatostatin receptor subtypes. Inactivation of sst(3) receptor function by heterodimerization with sst(2A). J Biol Chem. 2001; 276:14027-36.

36. Schulz S, Schreff M, Schmidt H, Händel M, Przewlocki R, Höllt V. Immunocytochemical localization of somatostatin receptor sst2A in the rat spinal cord and dorsal root ganglia. Eur J Neurosci. 1998; 10:3700-8.

37. Tweddle DA, Malcolm AJ, Bown N, Pearson AD, Lunec J. Evidence for the development of p53 mutations after cytotoxic therapy in a neuroblastoma cell line. Cancer Res. 2001; 61:8-13.
38. Kwok TT, Twentyman PR. The relationship between tumour geometry and the response of tumour cells to cytotoxic drugs-an in vitro study using EMT6 multicellular spheroids. Int J Cancer. 1985; 35:675-82.

39. Raggi CC, Maggi M, Renzi D, Calabrò A, Bagnoni ML, Scaruffi P, Tonini GP, Pazzagli M, De Bernardi B, Bernini G, Serio M, Orlando C. Quantitative determination of sst2 gene expression in neuroblastoma tumor predicts patient outcome. J Clin Endocrinol Metab. 2000; 85:3866-73.

40. Sestini R, Orlando C, Peri A, Tricario C, Pazzagli M, Serio M, Pagani A, Bussolati G, Granchi S, Maggi M. Quantitation of somatostatin receptor type 2 gene expression in neuroblastoma cell lines and primary tumors using competitive reverse transcription-polymerase chain reaction. Clin Cancer Res. 1996; 2:1757-65.

41. Watanabe N, Nakanishi Y, Kinukawa N, Ohni S, Obana Y, Nakazawa A, Nemoto N. Expressions of somatostatin receptor subtypes (SSTR-1, 2, 3, 4 and 5) in neuroblastic tumors; special reference to clinicopathological correlations with international neuroblastoma pathology classification and outcomes. Acta Histochem Cytochem. 2014; 47:219-29.

42. Zhang L, Vines DC, Scollard DA, McKee T, Konal T, Ganguly M, Do T, Wu B, Alexander N, Vali R, Shammas A, Besanger T, Baruchel S. Correlation of somatostatin receptor-2 expression with Gallium-68-DOTA-TATE uptake in neuroblastoma xenograft models. Contrast Media Mol Imaging. 2017; 2017:9481276. https://doi.org/10.1155/2017/9481276.

43. Csaba Z, Peineau S, Dournaud P. Molecular mechanisms of somatostatin receptor trafficking. J Mol Endocrinol. 2012; 48:R1-12.

44. Lesche S, Lehmann D, Nagel F, Schmid HA, Schulz S. Differential effects of octreotide and pasireotide on somatostatin receptor internalization and trafficking in vitro. J Clin Endocrinol Metab. 2009; 94:654-61.

45. Gleisner KS, Brolin G, Sundlöv A, Mjekiqi E, Östlund $\mathrm{K}$, Tennvall J, Larsson E. Long-term retention of $177 \mathrm{Lu} / 177 \mathrm{mLu}$-DOTATATE in patients investigated by $\gamma$-spectrometry and $\gamma$-camera imaging. J Nucl Med. 2015; 56:976-84.

46. Wang X, Tanaka M, Krstin S, Peixoto HS, Moura CC, Wink M. Cytoskeletal interference - A new mode of action for the anticancer drugs camptothecin and topotecan. Eur J Pharmacol. 2016; 789:265-74.

47. Giono LE, Manfredi JJ. The p53 tumor suppressor participates in multiple cell cycle checkpoints. J Cell Physiol. 2006; 209:13-20.

48. Tomicic MT, Christmann M, Kaina B. Topotecan-triggered degradation of topoisomerase $\mathrm{I}$ is p53-dependent and impacts cell survival. Cancer Res. 2005; 65:8920-6.

49. Tomicic MT, Christmann M, Kaina B. Topotecan triggers apoptosis in p53-deficient cells by forcing degradation of XIAP and survivin thereby activating caspase-3-mediated Bid cleavage. J Pharmacol Exp Ther. 2010; 332:316-25. 
50. Schmidt F, Rieger J, Wischhusen J, Naumann U, Weller M. Glioma cell sensitivity to topotecan: the role of p53 and topotecan-induced DNA damage. Eur J Pharmacol. 2001; 412:21-5.

51. Vivenza D, Monteverde M, Lattanzio L, Tonissi F, Astesana V, Denaro N, Comino A, Russi E, Lo Nigro C, Merlano M. Correlation of TP53 and MDM2 genotypes and clinical outcome in platinum-treated head and neck cancer patients with more than 10 years' follow-up. Int J Biol Markers. 2016; 31:e183-92.

52. Walter RF, Mairinger FD, Ting S, Vollbrecht C, Mairinger T, Theegarten D, Christoph DC, Schmid KW, Wohlschlaeger J. MDM2 is an important prognostic and predictive factor for platin-pemetrexed therapy in malignant pleural mesotheliomas and deregulation of P14/ARF (encoded by CDKN2A) seems to contribute to an MDM2-driven inactivation of P53. Br J Cancer. 2015; 112:883-90.

53. Liu W, He L, Ramirez J, Ratain MJ. Interactions between MDM2 and TP53 genetic alterations, and their impact on response to MDM2 inhibitors and other chemotherapeutic drugs in cancer cells. Clin Cancer Res. 2009; 15:7602-07.

54. Walsh EM, Niu M, Bergholz J, Xiao ZX. Nutlin-3 downregulates retinoblastoma protein expression and inhibits muscle cell differentiation. Biochem Biophys Res Commun. 2015; 461:293-9.

55. ClinicalTrials.gov. Available online: http://clinicaltrials.gov/ (accessed on 28 June 2017).

56. Wagner AJ, Banerji U, Mahipal A, Somaiah N, Hirsch H, Fancourt C, Johnson-Levonas AO, Lam R, Meister AK, Russo G, Knox CD, Rose S, Hong DS. Phase I trial of the human double minute 2 inhibitor MK-8242 in patients with advanced solid tumors. J Clin Oncol. 2017; 35:1304-11.
57. Yuan Y, Liao YM, Hsueh CT, Mirshahidi HR. Novel targeted therapeutics: inhibitors of MDM2, ALK and PARP. J Hematol Oncol. 2011; 4:16.

58. Graves B, Thompson T, Xia M, Janson C, Lukacs C, Deo D, Di Lello P, Fry D, Garvie C, Huang KS, Gao L, Tovar C, Lovey A, et al. Activation of the p53 pathway by smallmolecule-induced MDM2 and MDMX dimerization. Proc Natl Acad Sci USA. 2012; 109:11788-93.

59. Lu $\mathrm{X}$, Nannenga $\mathrm{B}$, Donehower LA. PPM1D dephosphorylates Chk1 and p53 and abrogates cell cycle checkpoints. Genes Dev. 2005; 19:1162-74.

60. Sriraman A, Radovanovic M, Wienken M, Najafova Z, Li Y, Dobbelstein M. Cooperation of Nutlin-3a and a Wip1 inhibitor to induce p53 activity. Oncotarget. 2016; 7:3162338. https://doi.org/10.18632/oncotarget.9302.

61. Chen Z, Wang L, Yao D, Yang T, Cao WM, Dou J, Pang JC, Guan S, Zhang H, Yu Y, Zhao Y, Wang Y, Xu X, et al. Wip1 inhibitor GSK2830371 inhibits neuroblastoma growth by inducing Chk2/p53-mediated apoptosis. Sci Rep. 2016; 6:38011.

62. Neshasteh-Riz A, Angerson WJ, Reeves JR, Smith G, Rampling R, Mairs RJ. Incorporation of iododeoxyuridine in multicellular glioma spheroids: implications for DNAtargeted radiotherapy using Auger electron emitters. Br J Cancer. 1997; 75:493-9.

63. Yuhas JM, Li AP, Martinez AO, Ladman AJ. Simplified method for production and growth of multicellular tumor spheroids. Cancer Res. 1977; 37:3639-43.

64. Ballangrud AM, Yang WH, Dnistrian A, Lampen NM, Sgouros G. Growth and characterization of LNCaP prostate cancer cell spheroids. Clin Cancer Res. 1999; 5:3171S-3176S. 